\title{
Numerical simulations of contrail-to-cirrus transition - Part 2: Impact of initial ice crystal number, radiation, stratification, secondary nucleation and layer depth
}

\author{
S. Unterstrasser and K. Gierens \\ Deutsches Zentrum für Luft- und Raumfahrt (DLR), Institut für Physik der Atmosphäre, Oberpfaffenhofen, Germany
}

Received: 18 June 2009 - Published in Atmos. Chem. Phys. Discuss.: 9 July 2009

Revised: 1 February 2010 - Accepted: 8 February 2010 - Published: 19 February 2010

\begin{abstract}
Simulations of contrail-to-cirrus transition were performed with an LES model. In Part 1 the impact of relative humidity, temperature and vertical wind shear was explored in a detailed parametric study. Here, we study atmospheric parameters like stratification and depth of the supersaturated layer and processes which may affect the contrail evolution. We consider contrails in various radiation scenarios herein defined by the season, time of day and the presence of lower-level cloudiness which controls the radiance incident on the contrail layer. Under suitable conditions, controlled by the radiation scenario and stratification, radiative heating lifts the contrail-cirrus and prolongs its lifetime. The potential of contrail-driven secondary nucleation is investigated. We consider homogeneous nucleation and heterogeneous nucleation of preactivated soot cores released from sublimated contrail ice crystals. In our model the contrail dynamics triggered by radiative heating does not suffice to force homogeneous freezing of ambient liquid aerosol particles. Furthermore, our model results suggest that heterogeneous nucleation of preactivated soot cores is unimportant. Contrail evolution is not controlled by the depth of the supersaturated layer as long as it exceeds roughly $500 \mathrm{~m}$. Deep fallstreaks however need thicker layers. A variation of the initial ice crystal number is effective during the whole evolution of a contrail. A cut of the soot particle emission by two orders of magnitude can reduce the contrail timescale by one hour and the optical thickness by a factor of 5 . Hence future engines with lower soot particle emissions could potentially lead to a reduction of the climate impact of aviation.
\end{abstract}

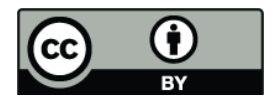

Correspondence to: S. Unterstrasser (simon.unterstrasser@dlr.de)

\section{Introduction}

In Part 1 (Unterstrasser and Gierens, 2009), a numerical model was introduced which is capable of performing an ample number of simulations of the transition of contrails into so-called contrail-cirrus. In that paper we studied mainly the influence of the meteorological conditions, i.e. relative humidity $\mathrm{RH}_{i}$, temperature $T$ and vertical wind shear $s$, on the transition, and we could demonstrate that the relative humidity is the most important environmental factor that determines contrail evolution and properties. But other factors, that could play a role or trigger interesting effects, have not yet been taken into consideration, and this will be done here. For instance radiation and its effect on the internal dynamics of a contrail will be studied for a number of "radiation scenarios". We will investigate how the stratification of the atmosphere affects the vertical displacement of radiatively heated contrails. Further, we test the hypothesis whether radiation can excite a strong contrail lifting that leads to homogeneous nucleation of crystals above the contrail. Similarly we assess whether heterogeneous nucleation of preactivated soot-cores (released from sublimated contrail ice crystals during the vortex phase) can be assumed to be a common process that increases the optical thickness of contrails in their later evolution. Moreover, the thickness of the supersaturated layer and the initial ice mass/number are varied, respectively, and the corresponding effect on the contrail-cirrus analysed. An overview over all simulations presented in this paper is given in Table 1 .

The paper is structured as follows: We begin with a brief description of the employed model in the following section. More details can be found in Part 1 . The various sensitivity studies are presented in Sects. 3 to 6. We discuss more

Published by Copernicus Publications on behalf of the European Geosciences Union. 
general implications of our results in Sect. 7 and draw conclusions in the final Sect. 8.

\section{Model setup}

As a detailed description of the model is given in Part 1, we will only sketch it here. We use EULAG to solve the momentum and thermodynamic equations (Smolarkiewicz and Margolin, 1997, 1998). Coupled to this is a microphysics module with a 2-moment-scheme (Spichtinger and Gierens, 2009) which solves prognostic equations for ice mass and number considering processes like ice deposition, sublimation and sedimentation. The crystal masses are assumed to be lognormally distributed in each grid box. Homogeneous/heterogeneous nucleation routines are switched on only if explicitly stated. The ice from different nucleation mechanisms and the contrail ice are stored in different ice classes. For each ice class the prognostic equations of the microphysical routine are solved simultaneously. This allows us to study the interaction of ice from different origins. For example we can study how the contrail evolution is impaired by surrounding natural cirrus or assess the potential that sublimated ice crystals re-nucleate. Quantities like ice number, ice mass, extinction and optical thickness of the total ice (i.e. consisting of several classes) are simply the sum of the specific quantities in the various classes. Since our computation of extinction requires a unimodal lognormal distribution of ice crystal mass, we note that the extinction coefficient $\chi_{m}$ is first calculated for each ice class reflecting their different lognormal distributions and then summed up. The index $m$ denotes the $m$-th ice class. In the same way the effective radius $r_{e, m}$ in each gridbox is calculated for each ice class separately. Similar to Part 1, Eq. (3), we use an extinction-weighted mean effective radius $\bar{r}_{e}$ :

$\bar{r}_{e}=\frac{\iint \sum_{m=1}^{\# \text { classes }} \chi_{m} r_{e, m} d x d z}{\iint \sum_{m=1}^{\# \text { classes }} \chi_{m} d x d z}$

In Part 1 all simulations dealt with steady ambient conditions, i.e. the ambient temperature and relative humidity did not change with time, as we did not consider synoptic-scale vertical motion $\left(w_{0}=0\right)$. In the present paper we test different nucleation processes and we allow a non-zero $w_{0}$. This updraught/downdraught is not explicitly resolved in the velocity field, but we use an external forcing term in the temperature equation to accommodate for the adiabatic temperature change:

$\frac{d \tilde{T}_{e}}{d t}=\frac{d T_{e}}{d t}+\Gamma_{d} w_{0}$

This formula is relevant only in the microphysics module, since in the dynamic core of EULAG the thermodynamic equation is written in terms of $\theta$ which is unaffected by adiabatic processes. $\frac{d T_{e}}{d t}$ is the uncorrected temperature forcing deduced from EULAG variables $\theta_{e}$ and $p_{e}$. The corrected temperature tendency $\frac{d \tilde{T}_{e}}{d t}$ includes the term $\Gamma_{d} w_{0}$ with $\Gamma_{d}=-g / c_{p}$ and vertical speed $w_{0}$ ( $>0$ for a updraught and $<0$ for a downdraught).

Moreover to study the radiation effect, a one-dimensional radiative transfer routine was implemented which will be explained in Sect. 4.1.

We run the model in 2 dimensions (vertical coordinate $z$ and horizontal coordinate $x$ normal to the flight direction) with resolution $\Delta x$ and $\Delta z \approx 10 \mathrm{~m}$. For initialisation we use the final microphysical fields of vortex phase simulations from Unterstrasser et al. (2008) (hereafter referred to as UGS08) They differ for the various relative humidities, temperatures and stabilities. The vortex-phase simulations were started with $\mathcal{N}_{00}^{*}=3.4 \cdot 10^{12} \mathrm{~m}^{-1}$ crystals and a variable fraction $f_{n}$ survives the vortex phase for the various ambient conditions. The number of crystals present at the beginning of the dispersion phase is $\mathcal{N}_{0}=\mathcal{N}_{00}^{*} \times f_{n}$.

Part 1 investigated the impact of temperature $T \in$ [209K, $222 \mathrm{~K}$ ], relative humidity with respect to ice $\mathrm{RH}_{i} \in$ $[105 \%, 130 \%]$ and vertical wind shear $s \in\left[0 \mathrm{~s}^{-1}, 6 \cdot 10^{-3} \mathrm{~s}^{-1}\right]$ on contrail-to-cirrus transition. Now the sensitivity of further parameters is studied at fixed triples $\left\{T, \mathrm{RH}_{i}, s\right\}$ and compared to the standard simulations.

For reasons of convenience we repeat a few definitions of Part 1, for details see there.

To determine average values of quantities like optical thickness, ice water content or ice crystal number concentration of a contrail, we use the definition of a predominant value $X_{\text {pre: }}$ :

$X_{\text {pre }}=\frac{\int_{A_{\text {sim }}} X^{2} d x d z}{\int_{A_{\text {sim }}} X d x d z}$,

where $X$ is a nonnegative quantity which tends to 0 outside the contrail area.

The total extinction $E$ is the horizontal integral of the extinction $1-e^{-\tau(x)}$ where $\tau(x)$ is the optical thickness of a column.

$E=\int\left(1-e^{-\tau}\right) d x \approx \int \tau d x=\iint \chi d x d z=\tilde{\tau} \times \tilde{B}$

with $\chi$ the extinction coefficient of the ice crystals. An advantage of this quantity is that the definition does not use any thresholds like the definitions of geometric properties. We define an intrinsic timescale of a contrail, which is the contrail age at which $E$ starts to decrease.

The vertical distribution of a contrail is studied evaluating vertical profiles of horizontally integrated extinction:

$\tau_{\text {hor }}(z)=\int \chi(x, z) d x$

This gives the optical thickness along a horizontal viewing direction (perpendicular to the flight direction).

For the sake of simplicity we use the word "contrail" over its total lifetime, although at later stages the term "contrailcirrus" would be more appropriate. In some studies we allow 
nucleation and the word "cirrus" is reserved for ice clouds formed naturally. From now on the ambient relative humidity is denoted as $\mathrm{RH}_{i}^{*}$ and is used to define the simulation setup. $\mathrm{RH}_{i}$ refers to the value at a specific time and location in the domain. Similarly, the temperature at cruise altitude $T_{\mathrm{CA}}$ is used to define a specific simulation run.

\section{Change of initial ice number and ice mass}

The number of ice crystals present at the beginning of the dispersion phase depends on meteorological and aircraft parameters. The number of ice crystals formed initially (during the first second after engine exit), $\mathcal{N}_{00}$, depends on the number of soot particles emitted from the engines, the temperature (relative to the threshold temperature for contrail formation) and the fuel sulphur content (Kärcher and Yu, 2009; Schumann et al., 2002; Kärcher et al., 1998; Schumann et al., 1996). Generally, the initial number of ice crystals is of the order $10^{12}$ to $10^{13} \mathrm{~m}^{-1}$. In our simulations of the vortexphase (UGS08) we used a standard value (marked by an asterisk) in the middle of that range, $\mathcal{N}_{00}^{*}=3.4 \cdot 10^{12} \mathrm{~m}^{-1}$.

During the vortex phase a variable fraction, $f_{n}$, of crystals sublimates in the primary wake depending mainly on relative humidity and temperature (UGS08; Huebsch and Lewellen, 2006). This fraction can be as low as 0.001 , but can also reach almost unity. All simulations of the dispersion phase in Part 1 have been initialised, according to temperature and relative humidity, with the respective $\mathcal{N}_{0}=\mathcal{N}_{00}^{*} \times f_{n}$. In this way we retain the effect of the wake processes on the later evolution of the contrail. Now our goal is to study the effect of varying initial conditions at contrail formation, i.e. varying $\mathcal{N}_{00}$. It turned out that $f_{n}$ does not much depend on $\mathcal{N}_{00}$ (UGS08), hence we can formulate our sensitivity study simply as simulations starting with $\mathcal{N}_{0}=\mathcal{N}_{00} \times f_{n}$, where we choose the non-standard $\mathcal{N}_{00}=\beta \times \mathcal{N}_{00}^{*}$ with the factor $\beta \in\{0.1,0.5,2,10\}$. Thus it suffices to multiply all ice number densities $N_{0}$ in the initial fields with $\beta$. The ambient conditions are $\mathrm{RH}_{i}^{*}=130 \%, T_{\mathrm{CA}}=222 \mathrm{~K}$ and $s=0 \mathrm{~s}^{-1}$.

The initial ice mass $\mathcal{I}_{0}$ is largely controlled by ambient conditions, hence we keep it unchanged for a first series of simulations. Of course, this results in changes of initial crystal sizes. In a second simulation series, both $I W C_{0}$ and $N_{0}$ are scaled simultaneously with $\beta$ which preserves the initial crystal sizes. Contrary to a variation of $\mathcal{N}_{0}$, an $\mathcal{I}_{0}$-modification over two orders of magnitude is rather an academic exercise. We see that the dominant ambient impact makes the initial perturbation of $I W C$ a short term effect on most contrail properties in the simulations (Fig. 1). Considering the predominant optical thickness, for example, the initial differences (solid to dotted lines) are forgotten after $1000 \mathrm{~s}$. One can notice a longer-lasting effect only for $I W C_{\text {pre }}$ and $\beta=0.1$ or 10 . But even in these unrealistic cases the initial perturbation vanishes within one hour. Fortunately, the model behaves as expected, and in the following we discuss only simulations with variation in $\mathcal{N}_{0}$.

The initial differences in $\mathcal{N}_{0}$ have a long memory. The initial scaling $\beta$ remains roughly present in $\mathcal{N}$ for the first three hours.

$$
\frac{\mathcal{N}(t, \beta)}{\mathcal{N}(t, \beta=1)} \approx \beta=\frac{\mathcal{N}(t=0, \beta)}{\mathcal{N}(t=0, \beta=1)}
$$

The differences in $\mathcal{N}$ have implications on many contrail properties. Clearly, the effective radii are smaller for higher $\mathcal{N}$.

The total extinction over the whole simulation time increases with initial ice crystal number. Both the evolution of total ice number and mass contribute to this result. First, the extinction of radiation is larger when crystals are smaller for a given $I W C$ and vice versa. Second, the total ice mass (not shown) gets larger when more crystals are present and vice versa, but not in the same proportion. For example a tenfold increase in total ice number may be accompanied by a doubling of the total ice mass. The increase of total ice mass is due to two mechanisms: during the first hours, higher crystal concentrations cause faster uptake of water vapour from entrained fresh air. Since contrail lateral dimensions are defined via their visibility, the spreading rate increases with the number of ice crystals present. At later stages, the sedimentation flux out of the contrail core region decreases with increasing number of ice crystals, because the fall speeds increase with crystal size. Moreover, this implies that the intrinsic timescale varies by roughly one hour when $\mathcal{N}_{0}$ is varied over two orders of magnitude.

The optical thickness increases for the total simulation time with initial ice crystal number. Again this is due to variations in number and mass concentrations. The ratio of $\tau_{\text {pre }}$ for two different $\mathcal{N}_{0}$-initialisations is nearly constant during the first three hours and can be expressed with the following formula.

$\tau_{\text {pre }}(t ; \beta) \approx \tau_{\text {pre }}(t ; \beta=1) \times \beta^{\gamma}$ with $\gamma=0.35$

For $t \geq 3 \mathrm{~h}$ the formula is no longer accurate since the contrail evolutions start to diverge due to differing sedimentation fluxes.

The diverging evolutions become also apparent in the vertical profiles of $\tau_{\text {hor }}$. After two hours all contrails still have a peak around $z=700 \mathrm{~m}$. The contrails with fewer ice crystals are located slightly lower. As the sedimentation flux is steadily larger, this leads to considerable differences becoming apparent after three hours. The contrail core is dried out for small $\mathcal{N}_{0}$ and the extinction in the fall streaks is as high as in the contrail core region. In contrast, sedimentation for large $\mathcal{N}_{0}$ is still so weak that the fallstreaks are not relevant relative to the contrail core. We carried out sensitivity studies for further ambient conditions and they all strongly resemble the above findings. 

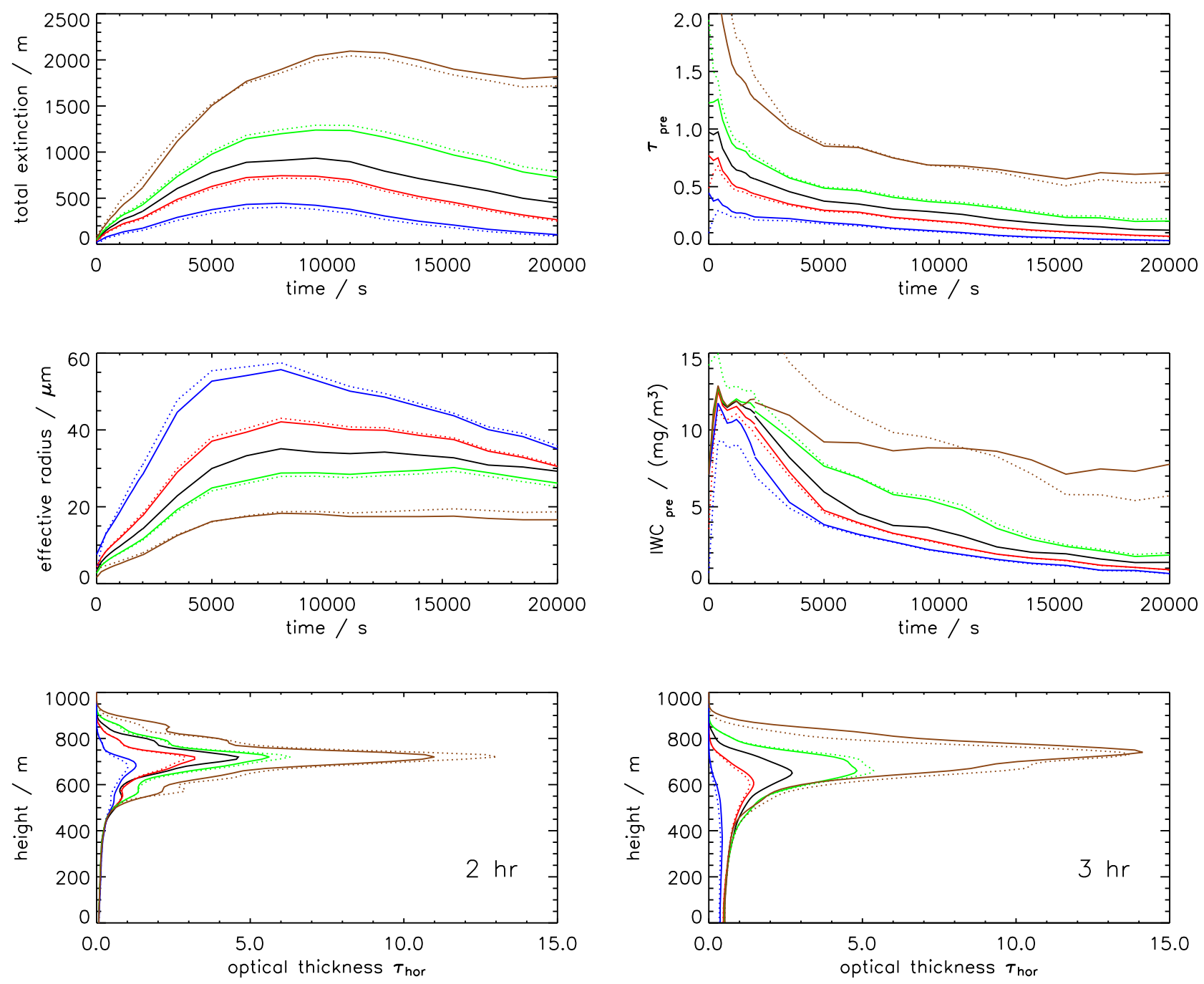

Fig. 1. Variation of initial ice number and ice mass for $T_{\mathrm{CA}}=222 \mathrm{~K}, \mathrm{RH}_{i}^{*}=130 \%$ and $s=0 \mathrm{~s}^{-1}$. The scaling factor $\beta$ is 0.1 (blue), 0.5 (red), 1 (standard simulation, black), 2 (green) and 10 (brown), respectively. Solid lines: only ice number was modified, dotted lines: both ice number and mass were modified. From top right to bottom left (row by row): total extinction, predominant optical thickness, effective radius, predominant ice water content, vertical profile of optical thickness $\tau_{\text {hor }}$ after 2 and $3 \mathrm{~h}$, respectively.

\section{Radiative impact}

\subsection{Radiation routine}

The ice particles in cirrus and contrails interact with solar and thermal radiation and change the radiative transfer by scattering, emission and absorption. Contrail dynamics is triggered by the gradient in the radiative heating/cooling rate (which is proportional to the Laplacian of the radiation flux density vector), in particular between the contrail and its environment. A warmer/colder contrail excites upward/downward convection and the temperature within the contrail then de/increases adiabatically. Depending on the stratification of the ambient air an adiabatic cooling can largely overcompensate the radiative warming and vice versa. Also the internal dynamics of a contrail can change due to differential heating/cooling. Generally, not only contrail dynamics gets affected by radiation, but also its microphysical properties since the excited convection changes the saturation pressure, hence the relative humidity distribution in the contrail.

To study the radiation-dynamics feedback, a onedimensional radiation routine after Fu and Liou (1993), Fu (1996) und Fu et al. (1998) was implemented in the EULAGcode. The radiation code was especially designed for fast radiative transfer computations of ice clouds and was recently employed in cirrus studies (Corti et al., 2006; Fusina et al., 2007).

The routine can handle ice crystals with effective radii between $r_{e}=6$ and $80 \mu m$. For smaller crystals, which can especially occur in young contrails, the parametrisation is 
not well-defined and leads to convergence problems. However, the radiative impact generally becomes apparent only over long timescales ( $\sim$ hours), over which the crystals easily grow beyond the critical lower limit. Two test studies showed that the ice crystals in gridboxes with $r_{e}<6 \mu \mathrm{m}$ can be neglected in the radiation routine. In a first run the ice mass is explicitly set to zero (where $r_{e}<6 \mu \mathrm{m}$ ) when passed to the radiation routine. The difference to a second run was negligible, where the ice mass was unchanged and $r_{e}$ was explicitly set to $6 \mu m$, when the actual $r_{e}$ was smaller. Although the radiative impact should be higher if we used the actual $r_{e}$-values, we believe that it is negligible. Additionally, we neglect the radiative effect of soot.

The heating rates inside the contrail depend on its microphysical properties (IWC and $r_{e}$ ) and on many ambient conditions which control the radiance incident on the contrail layer. We use vertical profiles of greenhouse gases $\left(\mathrm{H}_{2} \mathrm{O}\right.$, $\mathrm{CO}_{2}, \mathrm{O}_{3}, \mathrm{CH}_{4}, \mathrm{~N}_{2} \mathrm{O}$ ) and air temperature and density as input. Moreover, water clouds can be prescribed by setting liquid water content and droplet sizes. Anderson et al. (1986) supply typical vertical profiles in the range $z=0 \ldots 120 \mathrm{~km}$ for different latitudes and seasons. The background profiles are daily averages and are employed in a static sense. The time of day is set by changing the solar zenith angle. To simulate night conditions, the angle is set to $90^{\circ}$ and the incident solar radiation is zero.

The EULAG domain is $1-2 \mathrm{~km}$ deep and the bottom of the domain is shifted to an adequate height in the UTLS region such that the bottom EULAG temperature and the temperature of the background profile match. Outside the EULAG domain the vertical resolution of the given profiles is $1 \mathrm{~km}$. Inside the EULAG domain the fine resolution $\Delta z=5-10 \mathrm{~m}$ is kept and we use the EULAG values for temperature, density and water vapour. To avoid discontinuities at the top and bottom boundary, we attach $2 \mathrm{~km}$ thick interpolation layers with intermediate resolution where we linearly interpolate between the EULAG and the background values.

The radiation routine is called separately for each column of the model domain and computes vertical profiles of heating rates which serve as additional forcing $F_{R}$ in the thermodynamic equation in EULAG. It is assumed that the gain/loss of radiation energy of the crystals is instantaneously transferred to the ambient air and surface effects on the crystals are not taken into account. This omission is justified since the surface effect can be neglected for typical crystal sizes in contrails (Gierens, 1994) and showed no impact in previous contrail simulations (see sensitivity run 10 in Chlond, 1998).

After turning on the radiation routine a background cooling (up to $\sim-2 \mathrm{~K} / \mathrm{d}$ ) occurs in the cloudless regions of the EULAG domain for all simulations. The elevated water vapour concentrations (relative to drier background values above and below) lead to increased IR emission and thus the contrail layer is steadily cooled. This lowers the vapour saturation pressure and increases relative humidity. This is an undesired effect since ideally we would like to study the radiation affected contrail in the same environment as in the simulations without radiation in order to isolate the radiation effect on the contrail. Fortunately, a test showed that the interpretation of our results is practically not affected at all. We carried out a simulation where the contrail ice was set to zero in the radiation routine (i.e. the radiation does not interact with ice crystals) and the heating rates passed back to the EULAG main program contained only the background cooling. This simulation run is referred to as "radiation reference simulation" and the only difference to the usual standard simulation with turned off radiation routine is the consideration of the background cooling. The black dashed lines ("radiation reference simulation") in Fig. 4 show little difference to the standard simulation (black solid lines) and thus the impact of this background cooling on the contrail dynamics and properties is negligible.

\subsection{Radiative impact on contrail evolution}

\subsubsection{Variation of the radiation scenario}

The impact of radiation on contrail evolution is studied for different seasons, times of day and lower-level cloudiness. These three parameters determine what we call the "radiation scenario". For the different seasons we use typical atmospheric background profiles for midlatitude winter/summer conditions. The daytime is given by solar zenith angle $\left(45^{\circ}\right.$ at day, $90^{\circ}$ at night). To study the effect of lower-level cloudiness, we prescribe a water cloud with constant $L W C=1 \mathrm{~g} / \mathrm{m}^{3}$ between $z=4$ and $6 \mathrm{~km}$. All simulations used a surface albedo value of $a_{\mathrm{sfc}}=0.05$. A sensitivity run with $a_{\mathrm{sfc}}=0.3$ revealed a weak sensitivity to this parameter and thus it is not mentionned in the following discussion.

Generally we study the radiative impact at ambient conditions $\left(T_{\mathrm{CA}}=217 \mathrm{~K}, \mathrm{RH}_{i}^{*}=130 \%\right.$ and $\left.s=0 \mathrm{~s}^{-1}\right)$ which lead to large, long-living contrails. For this parameter choice the radiative impact is likely to be most pronounced (within the studied parameter range for $T_{\mathrm{CA}}, \mathrm{RH}_{i}^{*}$ and $s$ ). Contrails at lower relative humidity have smaller ice mass (concentrations) and the heating rates are smaller (see Table 1 for an overview of simulations discussed in this paper). We assume a stability of the atmosphere of $N_{\mathrm{BV}}=1 \cdot 10^{-2} \mathrm{~s}^{-1}$. This is an important parameter since it controls the vertical displacement of a contrail for a fixed heating rate. In the next section the sensitivity to $N_{\mathrm{BV}}$ is discussed.

Figure 2 shows the heating rate inside the model domain at $t=6500 \mathrm{~s}$ for summer day conditions with no water cloud below. The position of the contrail is sketched with $I W C=2$ and $6 \mathrm{mg} / \mathrm{m}^{3}$-isolines. Outside the contrail the heating rates are slightly negative as stated earlier. Inside the contrail they exceed $15 \mathrm{~K} / \mathrm{d}$, especially at spots with high $I W C$. The heating is spatially inhomogeneous. This leads to a stretching of the contrail in the vertical direction since the top layer rises faster than the underlying layers. 


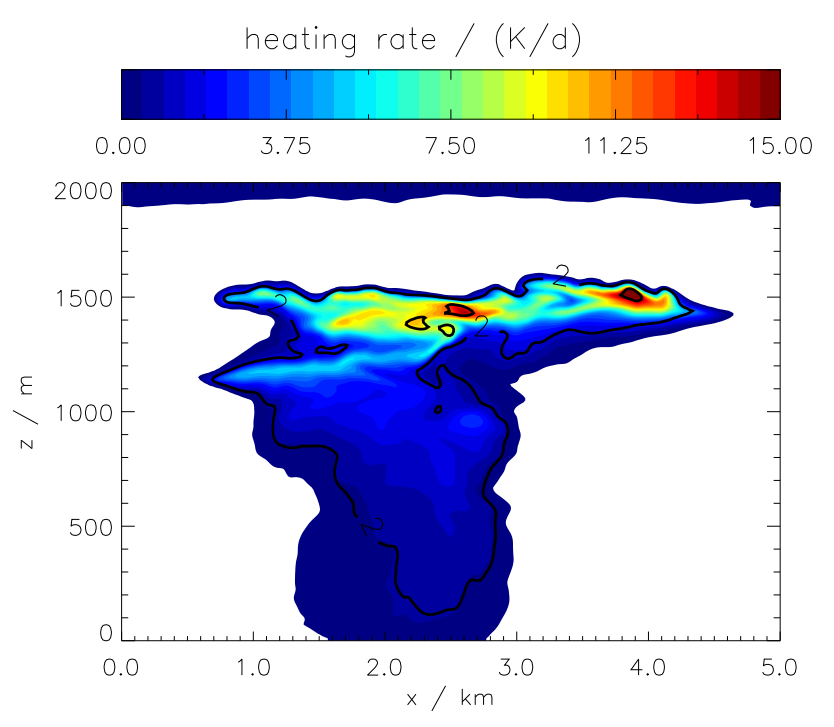

Fig. 2. Spatial distribution of the heating rate for $t=6500 \mathrm{~s}, T_{\mathrm{CA}}=217 \mathrm{~K}, \mathrm{RH}_{i}^{*}=130 \%$ and $s=0 \mathrm{~s}^{-1}$ on a cloud-free summer day. Negative values in the white area. For orientation, $I W C=2 \mathrm{mg} / \mathrm{m}^{3}$ and $6 \mathrm{mg} / \mathrm{m}^{3}-$ isolines are added. The original flight level is at $z=1300 \mathrm{~m}$.

Figure 3 shows vertical profiles of mean differential heating rates inside the contrail. First, the differential heating rates are computed by simply subtracting the background cooling everywhere. Then the heating rates are averaged over the contrail area. Only gridboxes with $I W C>0.5 \mathrm{mg} / \mathrm{m}^{3}$ are considered. These profiles allow an intercomparison of the different radiation scenarios and their effect on contrail evolution. With a cloudless atmosphere below, a contrail is heated for all studied seasons and day-times. The strongest heating rates occur during summer, especially during the day. Then the contrails are mostly heated in the infrared spectrum. During the day the sun additionally heats in the shortwave range ( $\sim+30 \%$ of IR heating). During a winter day the IR and solar radiation contribute roughly equally to the contrail heating. During a winter night the IR heating alone is too weak to excite an updraught. The profile of $\tau_{\text {hor }}$ shows that the simulated contrails have risen by up to $300 \mathrm{~m}$ within the first two hours compared to cases without contrail heating (standard simulation without radiation and "radiation reference simulation", black lines). In winter scenarios the uplift is smaller than in summer scenarios.

Generally the radiative impact is much less pronounced when there is a water cloud at a lower level (not shown). The contrail receives less IR radiation from below since the water cloud emits at a lower temperature than the ground. In our simulations this leads to a cooling in the IR which may be roughly compensated by a heating in the solar spectrum. During night and/or winter the presence of a water cloud leads to a slight cooling (IR + solar) of the contrail and a faster disappearance during its subsidence. Only during a summer day the contrails are slightly heated when there is a cloud beneath.

The triggered vertical motion affects the microphysical and geometric properties. Basically an updraught leads to a higher total extinction since the ice water content grows, whereas contrail ice mass sublimates during a downdraught. Figure 4 shows the temporal evolution of total extinction, now additionally displaying cases with an underlying water cloud in the right panels. Scenarios without a water cloud develop differently from the respective standard simulation after $1-3 \mathrm{~h}$. While the contrail gets weaker (in terms of $E$ ) in the standard simulation because of sedimentation, this effect is (partly) compensated by the uplifting in the radiation cases. Hence radiation can prolong the lifetime of contrails, especially during summer and to a lesser extent during winter. As the figure shows, the radiation effect on $E$ is large under cloudless summer conditions and for the selected stability. The cloudless winter and all cloudy cases show also a significant radiative impact which can nevertheless also be achieved by small variations (a few percent) of the background relative humidity (cf. Part 1, Sect. 3.3.6). In the cloudy cases the deviations of $E$ from the standard simulation with no radiation are generally smaller and around $\pm 20 \%$.

In a further test run (not shown) we increased the temperature to $T_{\mathrm{CA}}=222 \mathrm{~K}$. The increased ice mass leads to a stronger radiative impact. The reduction of the temperature difference between the contrail and the earth's surface or an underlying cloud, respectively, turned out to be a second order effect.

Finally, we conclude that the radiation scenario has an impact on the contrail evolution which depends most sensitively on the existence of a cloud underneath, followed by the season and the time of day. Most favourable conditions (in terms of higher resulting $E$-values) for the contrail evolution are given on cloudless summer days, the least favourable during cloudy winter nights.

\subsubsection{Variation of stratification}

As seen above radiation alters the internal dynamics of the contrail. The heating rates depend on the radiation scenario which determines the radiation flux entering the contrail layer and on the microphysical properties of the contrail which determine how much of the radiation is converted to heat and kinetic energy. The magnitude of the vertical displacement depends on the stratification. In the preceding section the impact of the radiation scenario was investigated. Now the impact of stratification is studied. The sensitivity study was carried out for Brunt-Väisälä frequencies $N_{\mathrm{BV}}=0.5,0.7,1.0,1.3$ and $2 \cdot 10^{-2} \mathrm{~s}^{-1}$. The ambient conditions are $T_{\mathrm{CA}}=217 \mathrm{~K}, \mathrm{RH}_{i}^{*}=120 \%$ and $s=0 \mathrm{~s}^{-1}$. The radiation scenario was chosen such that we expect a maximal updraught (cloudless summer day). Especially in the weakly stable cases, the height of the simulation domain was 

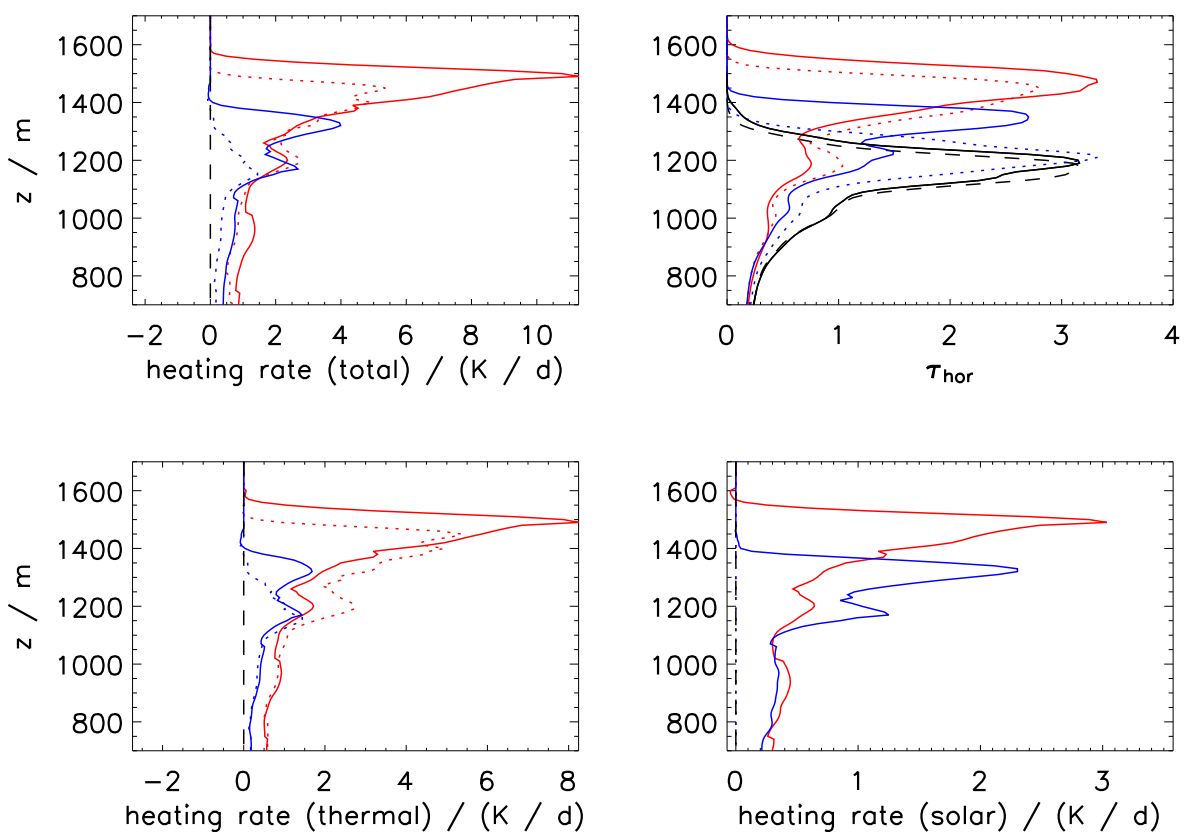

Fig. 3. Vertical profiles of mean heating rates inside the contrail minus background heating rates (total: top-left; thermal: bottom-left; solar: bottom-right) and optical thickness $\tau_{\text {hor }}$ (top-right) at $t=6500$ s for different radiation scenarios: summer/winter (red/blue) and day/night (solid/dotted). The $\tau_{\text {hor }}$-figure additionally shows the profiles of the standard simulation with no radiation (black, solid) and the radiation reference simulation (black, dashed). No heating rates are shown since in both simulations no differential contrail heating occurs. The ambient conditions are $T_{\mathrm{CA}}=217 \mathrm{~K}, \mathrm{RH}_{i}^{*}=130 \%$ and $s=0 \mathrm{~s}^{-1}$. The original flight level is at $z=1300 \mathrm{~m}$.
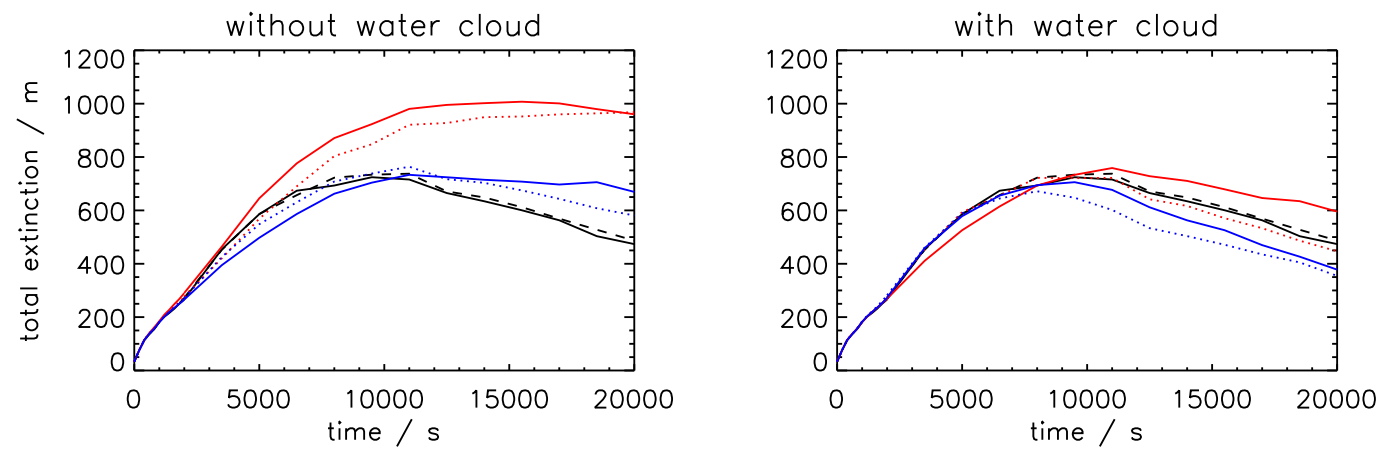

Fig. 4. Temporal evolution of total extinction $E$ for different radiation scenarios: without/with water cloud (left/right), summer/winter (red/blue) and day/night (solid/dotted). For comparison: standard simulation with no radiation (black, solid) and radiation reference simulation (black, dashed). The ambient conditions are $T_{\mathrm{CA}}=217 \mathrm{~K}, \mathrm{RH}_{i}^{*}=130 \%, s=0 \mathrm{~s}^{-1}$ and $N_{\mathrm{BV}}=1 \cdot 10^{-2} \mathrm{~s}^{-1}$.

enlarged up to $L_{z}=5 \mathrm{~km}$, such that the contrail is always far away from the upper boundary.

Stratification affects the contrail evolution via three ways. First, the initialisation of the microphysical fields differs from case to case since stratification affects the contrail evolution already during the vortex phase. The more stable the atmosphere, the faster the vortices decay, which leads to a smaller vertical extent and more crystals present at the end of the vortex phase. Between $36 \%$ and $61 \%$ of the initial crystals survive the vortex phase for the chosen ambient conditions. Second, the turbulent flow field depends on stratification, e.g. the ratio of vertical and horizontal eddy diffusivity is higher in a weakly stratified atmosphere and vice versa. Third, the aforementioned radiation effect depends on stratification, as we will see.

In a first step the radiation is turned off to better discriminate between the various effects in the analysis. In a second step we turned on the radiation routine. Besides simply quantifying the stratification impact a further motivation was to check whether the updraught can lead to nucleation of new particles, as suggested in Jensen et al. (1998). Thus the homogeneous nucleation routine is now activated in the 
Table 1. List of sensitivity simulations. The type of sensitivity study is given in the left column. The middle column contains a more precise description of each sensitivity study. The standard parameter value is marked by a box. The right column denotes the relative humidity, temperature and wind shear at which the sensitivity was studied. The letter " $\mathrm{R}$ " indicates that radiation is turned on and the letter " $\mathrm{S}$ " indicates that a cloudless summer day was used as radiation scenario. Parameter combinations printed in boldface are discussed in the paper in more detail than the others. In simulations with synoptic up/downdraught the background relative humidity and temperature varies with time (as indicated by the arrows $\nearrow$ and $\searrow$ ).

\begin{tabular}{|c|c|c|}
\hline $\begin{array}{l}\text { Sensitivity } \\
\text { parameter }\end{array}$ & variability & $\begin{array}{l}\text { runs at }\left(R H_{i}^{*} / \%\right. \\
\left.T_{\mathrm{CA}} / \mathrm{K}, s /\left(10^{-3} \mathrm{~s}^{-1}\right)\right)\end{array}$ \\
\hline ice crystal number & $\mathcal{N}_{00}=\beta \times \mathcal{N}_{00}^{*}$ with $\beta \in\{0.1,0.5,1,2,10\}$ & $(\mathbf{1 3 0 , 2 2 2 , 0}),(130,222,6)$ \\
\hline ice crystal number and mass & $\mathcal{N}_{00}=\beta \times \mathcal{N}_{00}^{*}$ and $\mathcal{I}_{00}=\beta \times \mathcal{I}_{00}^{*}$ with $\beta \in\{0.1,0.5,1,2,10\}$ & $(\mathbf{1 3 0}, \mathbf{2 2 2}, \mathbf{0}),(130,222,6)$ \\
\hline \multirow[t]{2}{*}{ radiation scenario } & 8 runs determined by season (summer/winter), time of day (day/night) and lower-level cloudiness (yes/no) & $(130,217,0) \mathrm{R}$ \\
\hline & $\begin{array}{l}\text { albedo } a_{\mathrm{sfc}}=0.3 \text { instead of default value } 0.05 \\
\text { further simulation at various ambient conditions }\end{array}$ & $\begin{array}{c}(130,217,0) \mathrm{RS} \\
(130,222,0) \mathrm{RS} \\
(130,212,0) \mathrm{RS} \\
(120,217,0) \mathrm{RS} \\
(110,217,0) \mathrm{RS}\end{array}$ \\
\hline \multirow[t]{4}{*}{$\begin{array}{l}\text { stratification and radia- } \\
\text { tion-induced homoge- } \\
\text { neous nucleation }\end{array}$} & $N_{\mathrm{BV}} \in\{0.5,0.7,1.0,1.3,2\} \times 10^{-2} \mathrm{~s}^{-1}$ & $\begin{array}{l}(120,217,0) \\
(120,217,0) R S \\
(120,217,10) R S\end{array}$ \\
\hline & $N_{\mathrm{BV}}=0.7 \times 10^{-2} \mathrm{~s}^{-1}$ & $\begin{array}{l}(130,217,4) \mathrm{RS} \\
(130,217,4) \mathrm{RS}\end{array}$ \\
\hline & $N_{\mathrm{BV}}=1.0 \times 10^{-2} \mathrm{~s}^{-1}$ & $\begin{array}{l}(140,222,0) \mathrm{RS} \\
(140,222,10) \mathrm{RS}\end{array}$ \\
\hline & $N_{\mathrm{BV}}=1.0 \times 10^{-2} \mathrm{~s}^{-1}$ and solar zenith angle $=0^{\circ}$ & $(130,217,0) \mathrm{RS}$ \\
\hline \multirow[t]{2}{*}{ supersaturated layer } & $L_{\mathrm{RH}_{i}^{*}} \in\{1 \mathrm{~km}, 800 \mathrm{~m}, 600 \mathrm{~m}, 400 \mathrm{~m}\}$ & $(120,217,0)$ \\
\hline & & $(120,217,6)$ \\
\hline \multicolumn{3}{|l|}{ re-nucleation } \\
\hline \multirow[t]{2}{*}{ reference run } & $\begin{array}{l}w_{0}=10 \mathrm{cms}^{-1} \text { for } t \in[1000 \mathrm{~s}, 3000 \mathrm{~s}] \text { and } \\
w_{0}=-10 \mathrm{cms}^{-1} \text { for } t \in[4000 \mathrm{~s}, 6000 \mathrm{~s}]\end{array}$ & $\begin{array}{l}(120 \nearrow 152 \searrow 120 \\
217 \searrow 215 \nearrow 217,0)\end{array}$ \\
\hline & $\begin{array}{l}\text { like reference run and heterogeneous nucleation with } \\
R H_{\text {het }} \in\{101 \%, 105 \%, 110 \%, 120 \%, 130 \%\}\end{array}$ & $\begin{array}{l}(120 \nearrow 152 \searrow 120 \\
217 \searrow 215 \nearrow 217,0)\end{array}$ \\
\hline \multirow[t]{2}{*}{ reference run $R A D$} & like reference run + radiation & $\begin{array}{l}(120 \nearrow 152 \searrow 120, \\
217 \searrow 215 \nearrow 217,0) R S\end{array}$ \\
\hline & $\begin{array}{l}\text { like reference run } R A D \text { and heterogeneous nucleation with } \\
\mathrm{RH}_{\mathrm{het}}=120 \%\end{array}$ & $\begin{array}{l}(120 \nearrow 152 \searrow 120, \\
217 \searrow 215 \nearrow 217,0) R S\end{array}$ \\
\hline
\end{tabular}

microphysical module. Most likely nucleation should occur, if at all, above and below the ascending contrail where no crystals are present and the relative humidity could steadily rise beyond the nucleation threshold. Inside the contrail core the relative humidity is around saturation and one cannot expect nucleation there, since newly available water vapour deposits on the crystals within short timescales. How much crystal-free air is lifted depends also on the shape/width of the contrail. Thus in a further simulation series the vertical wind shear was set to $s=10 \cdot 10^{-3} \mathrm{~s}^{-1}$ to study very broad contrails. An overview over the simulations presented in this section is given in Table 1.

In a weakly stable atmosphere with $N_{\mathrm{BV}}=0.5 \cdot 10^{-2} \mathrm{~s}^{-1}$ the simulations show strong turbulent fluctuations in the vertical direction (not shown). In all 3 cases (Table 1) the relative humidity overshoots the nucleation threshold in the whole simulation domain independently of the contrail. A cirrus with its characteristic patchy structure forms in the simulation area but nevertheless detached from the contrail reducing the relative humidity in the contrail neighbourhood. At later stages this constrains lateral spreading of the contrail. As this cirrus formation is not connected with the contrail dynamics, it is not of interest for our investigation; hence a more detailed discussion of the interaction of contrails and natural cirrus in their environment is not in the scope of the present paper. In Fig. 5 the total extinction is shown for the simulations with $s=0 \mathrm{~s}^{-1}$. With radiation switched off, the sensitivity of contrail development on stratification is low. The contrail's $E$ and lifetime increase slightly with stratification. Also the initial crystal number (after the vortex phase) increases with stratification: For $N_{\mathrm{BV}}=2.0 \cdot 10^{-2} \mathrm{~s}^{-1}$ it is approximately two times that for $N_{\mathrm{BV}}=0.5 \cdot 10^{-2} \mathrm{~s}^{-1}$. Hence the sedimentation flux decreases with increasing stratification (see $\mathcal{N}_{0}$-sensitivity in Sect. 3). With radiation switched on, the stratification impacts on these properties are opposite and much larger variation results. The total extinction is the 
larger the less stable the atmosphere is. In the strongly stable case $\left(N_{\mathrm{BV}}=2.0 \cdot 10^{-2} \mathrm{~s}^{-1}\right)$, radiation has only a small impact, since the vertical displacement due to contrail heating is negligible (see Fig. 6). Radiation strongly affects the contrail evolution in slightly stable conditions and the total extinction is two to three times higher than under strongly stable conditions. Liu et al. (2003) studied the radiative impact on natural cirrus and they found consistent with our results that only in a conditionally unstable atmosphere the cirrus evolution changes qualitatively.

In radiation scenarios in which contrails are cooled, radiation would lead to a faster disappearance (not shown).

The additional dynamics triggered by radiation and weak stratification did not suffice in our model to cause nucleation of ice in the contrail's environment, neither above nor below the contrail. In case of a narrow contrail $\left(s=0 \mathrm{~s}^{-1}\right)$ the lifted air flows out horizontally and the humidity increase is not substantial. In a strongly sheared environment the contrails are much broader and have lower ice mass concentrations. Hence less radiation energy is absorbed and converted per contrail unit width and the heating rates are thus small. The vertical displacement of such a contrail is not sufficient to lift the relative humidity above the nucleation threshold (see Fig. 6). In order to test the latter statement we even simulated cases with still higher relative humidities (up to 140\%) and higher temperatures $(222 \mathrm{~K})$, and with medium wind shear $\left(4 \cdot 10^{-3} \mathrm{~s}^{-1}\right)$ which should neither lead to too low $I W C$ nor to too narrow contrails (see Table 1 for list of simulations). We also increased the incident solar radiation flux by setting the solar zenith angle to zero. In no case we were able to find homogeneous nucleation close to the lifting contrails that was characteristically different from the corresponding ice formation further away. Hence we conclude that radiation-induced homogeneous nucleation is not relevant for a very wide range of ambient conditions.

In contrast to our results, Jensen et al. (1998) found in simulations with ambient $\mathrm{RH}_{i}=125 \%$ humidities directly above the lifting contrail that would suffice for homogeneous nucleation. In the rest of their domain the humidity was nearly constant around the initial value, hence far below the nucleation threshold. As their initial parameters $\left(\mathrm{RH}_{i}=125 \%\right.$, $T_{\mathrm{CA}}=222 \mathrm{~K}, s=6 \cdot 10^{-3} \mathrm{~s}^{-1}$ and $d T / d z=-9.5 \mathrm{~K} \mathrm{~km}^{-1}$ ) were similar to ours, these cannot make the difference. Instead we believe that the spatial structure of the turbulent wind field is the main cause of the differences. The humidity field outside of the contrail (Fig. 4 in Jensen et al., 1998) is very smooth, and the vertical wind field in their domain mainly has one large uplift region around the contrail and downdraught regions elsewhere. This strongly suggests that the initial random perturbations in their model became quickly (i.e. within the first hour) damped away. Quick damping of random wind fluctuations is a symptom of uncorrelated initial perturbations. In contrast, the initial fluctuations in our model are autocorrelated, hence their damping time scale is much longer than one hour. In effect, the radiation-induced

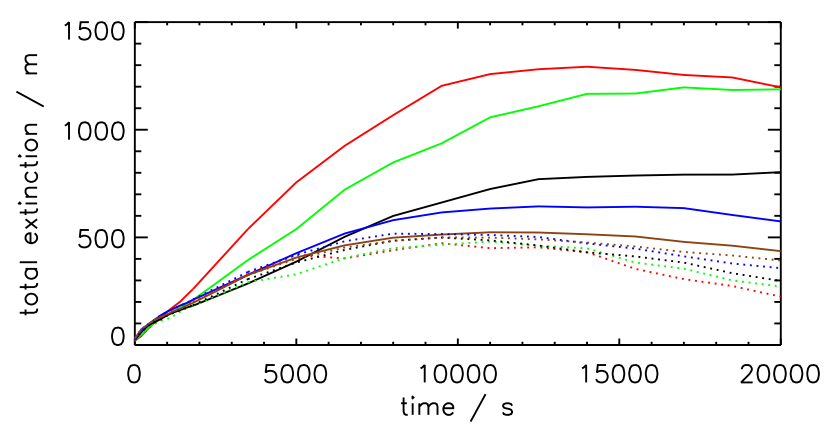

Fig. 5. Temporal evolution of total extinction $E$ for different stratification. $\quad N_{\mathrm{BV}} /\left(10^{-2} \mathrm{~s}^{-1}\right)=0.5$ (red), 0.7 (green), 1.0 (black), 1.3 (blue) and 2.0 (brown). The radiation routine is turned on (solid) or off (dotted). The ambient conditions are $T_{\mathrm{CA}}=217 \mathrm{~K}$, $\mathrm{RH}_{i}^{*}=120 \%, s=0 \mathrm{~s}^{-1}$.

uplift of the contrail has a smaller magnitude than the turbulent velocity fluctuations. These wind fluctuations lead to strong fluctuations in the humidity field and these are similar above and far away from the contrail.

\section{Depth of the supersaturated layer}

In this section we vary the thickness of the supersaturated layer. In the base case a $L_{\mathrm{RH}_{i}^{*}}=1 \mathrm{~km}$ deep layer with constant $\mathrm{RH}_{i}^{*}$ was prescribed. Together with the $500 \mathrm{~m}$ thick transition zones above and below that layer we get a $\sim 1.3 \mathrm{~km}$-deep supersaturated layer for $\mathrm{RH}_{i}^{*}=120 \%$. About $1 \mathrm{~km}$ of the layer is below the original flight level, as shown in Fig. 7. This figure also displays the humidity profiles of further simulations with $L_{\mathrm{RH}_{i}^{*}}=800 \mathrm{~m}, 600 \mathrm{~m}$ and $400 \mathrm{~m}$. As the primary wake sinks at most $300 \mathrm{~m}$ below the flight level, the initial contrail ice lies in all cases completely inside the supersaturated region. We do not treat cases where the primary wake sinks into a subsaturated layer during the vortex phase. The ambient conditions are $T_{\mathrm{CA}}=217 \mathrm{~K}, \mathrm{RH}_{i}^{*}=120 \%, s=0 \mathrm{~s}^{-1}$. Figure 8 shows vertical profiles of $\tau_{\text {hor. }}$. After 2000 s no fallstreaks have formed yet and the profiles are nearly identical for the various $L_{\mathrm{RH}_{i}^{*}}$. After $t=6500 \mathrm{~s}$ and $11000 \mathrm{~s}$, the fallstreaks are smaller for $L_{\mathrm{RH}_{i}^{*}}=600 \mathrm{~m}$ and especially $400 \mathrm{~m}$. Nevertheless, the core region is virtually unaffected. Obviously, a thinner supersaturated layer does not lead to a weakening of the core region, it merely reduces the depth of the fallstreaks. As most crystals are located in the core region and only a small fraction sediments (less than $5 \%$ over $6 \mathrm{~h}$ ), the fraction of sublimating crystals is similar for the various $L_{\mathrm{RH}_{i}^{*}}$ (not shown). The above findings are consistent with the results of Part 1 where the standard simulations already showed that vertical growth is due to sedimentation and to a lesser extent due to turbulent mixing and that the core region contains a large fraction of the crystals and stays at about the same altitude (when the radiation routine is turned off). 

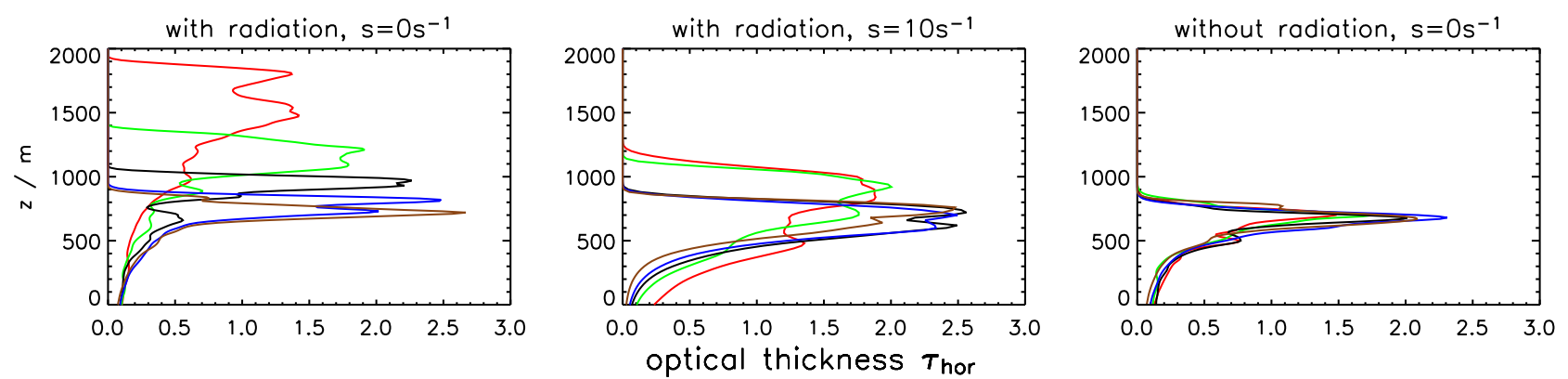

Fig. 6. Vertical profile of optical thickness $\tau_{\text {hor }}$ for different stratification at $t=6500 \mathrm{~s} . N_{\mathrm{BV}} /\left(10^{-2} \mathrm{~s}^{-1}\right)=0.5$ (red), 0.7 (green), 1.0 (black), 1.3 (blue) and 2.0 (brown). The activation of the radiation routine and the wind shear are indicated above each column. The ambient conditions are $T_{\mathrm{CA}}=217 \mathrm{~K}, \mathrm{RH}_{i}^{*}=120 \%$. The original flight level is at $z=800 \mathrm{~m}$.
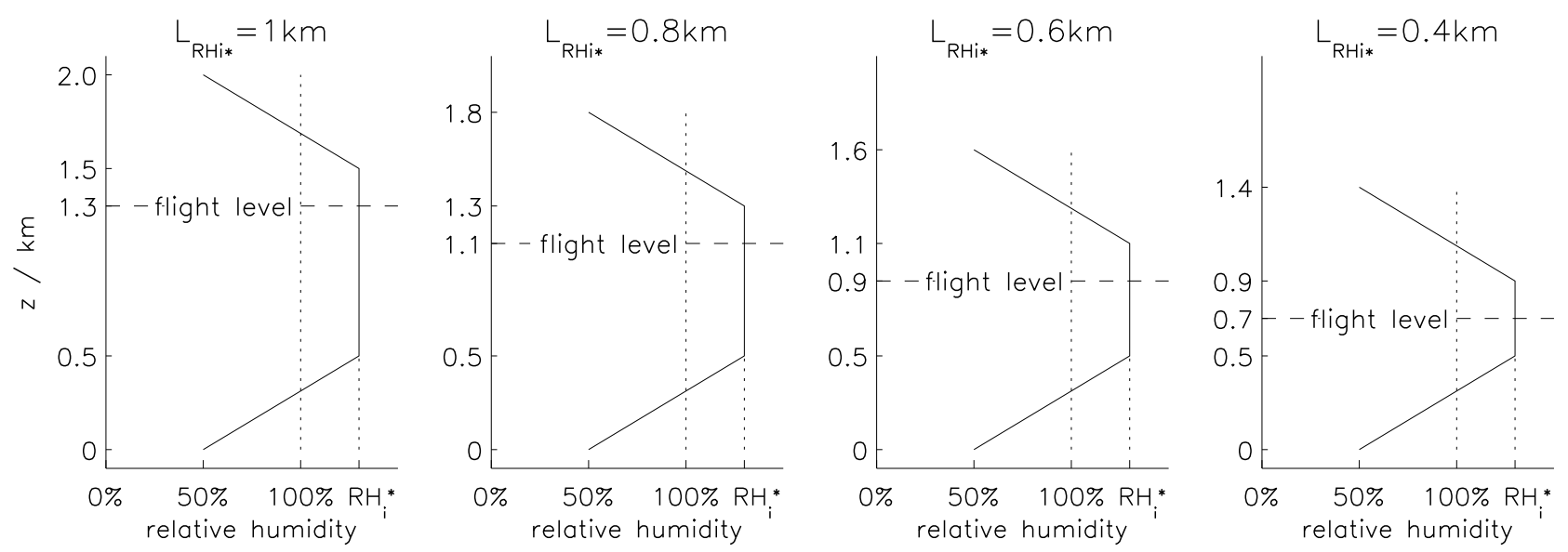

Fig. 7. Vertical profiles of background relative humidity with various thicknesses of the supersaturated layer.

The importance of a fallstreak increases with time as its ice mass increases. More and larger ice crystals sediment out of the core region. The ice mass of the core region starts to diminish as soon as the sedimentation loss is no longer balanced by depositional growth. At later stages in the contrailcirrus evolution the ice in the fallstreaks contributes substantially to the optical thickness (along the vertical) and to the total extinction $E$. Figure 9 shows the temporal evolution of total extinction and optical thickness (along the vertical). The total extinction is initially nearly identical for the various $L_{\mathrm{RH}_{i}^{*}}$. However, $E$ increases longer and attains larger maxima the deeper the supersaturated layer is. The intrinsic timescale of the contrails rises from two $\left(L_{\mathrm{RH}_{i}^{*}}=400 \mathrm{~m}\right)$ to three $\left(L_{\mathrm{RH}^{*}}=1 \mathrm{~km}\right)$ hours. Analogously the optical thickness is larger when the fallstreaks are vertically more extended.

Concluding, it seems that contrails are persistent and can evolve substantially in supersaturated layers of $\gtrsim 500 \mathrm{~m}$ depth. Radiation might cause a contrail to move out of a thin layer. However, if radiation causes an updraught, the contrail layer adiabatically cools and supports contrail growth as excess water vapour becomes available for deposition. Then it also depends on the humidity profile above the supersaturated layer whether the contrail can grow on once it gets lifted above that layer.

\section{Potential of re-nucleation}

Many ice crystals sublimate already during the vortex phase and their soot cores stay within the contrail region or in its immediate environment. These soot particles may act as centres for heterogeneous ice nucleation during the contrail-tocirrus transformation phase, in particular since pre-activation inside the former ice crystals might have made them potent ice nuclei with relatively low nucleation threshold. The potential of these soot particles to re-nucleate ice during the later phases of contrail development is assessed in this section. (Aircraft combustor soot may also be able to affect ice cloud formation far downstream and long after its emission; these effects are not studied here.)

We assume that each ice crystal that sublimates during the vortex phase releases one pre-activated soot particle. Although it is not known whether pre-activation occurs at all, we assume - in the spirit of a sensitivity study 

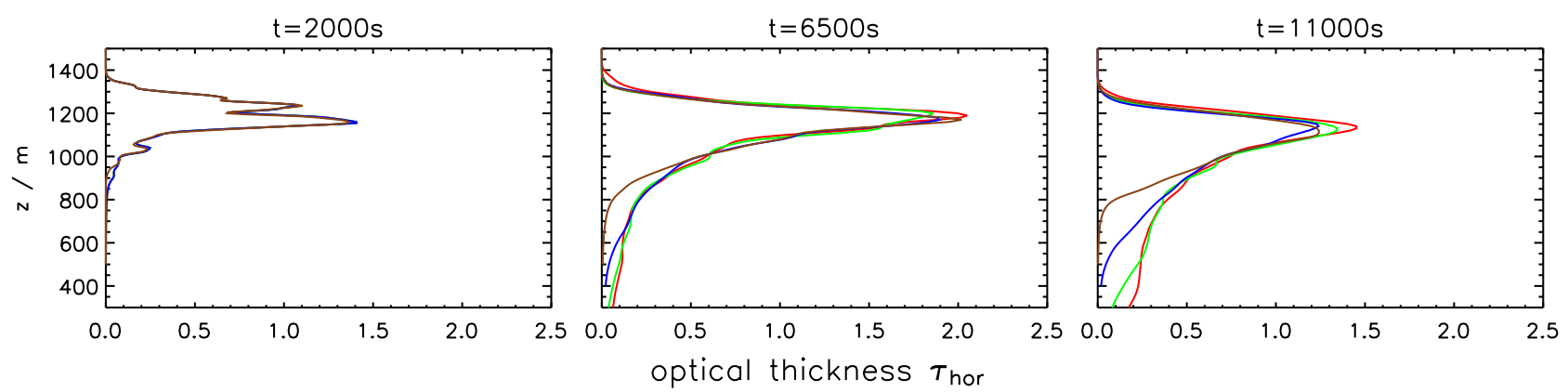

Fig. 8. Vertical profiles of optical thickness $\tau_{\text {hor }}$ at $t=2000 \mathrm{~s}, 6500 \mathrm{~s}$ and $11000 \mathrm{~s}$ for different thicknesses $L_{\mathrm{RH}}^{*}$ of the supersaturated layer. $L_{\mathrm{RH}_{i}^{*}}$ is $1 \mathrm{~km}$ (red), $800 \mathrm{~m}$ (green), $600 \mathrm{~m}$ (blue) resp. $400 \mathrm{~m}$ (brown). The ambient conditions are $T_{\mathrm{CA}}=217 \mathrm{~K}, \mathrm{RH}_{i}^{*}=120 \%$ and $s=0 \mathrm{~s}^{-1}$. The profiles were shifted in the vertical such that the original flight level is always at $z=1300 \mathrm{~m}$.
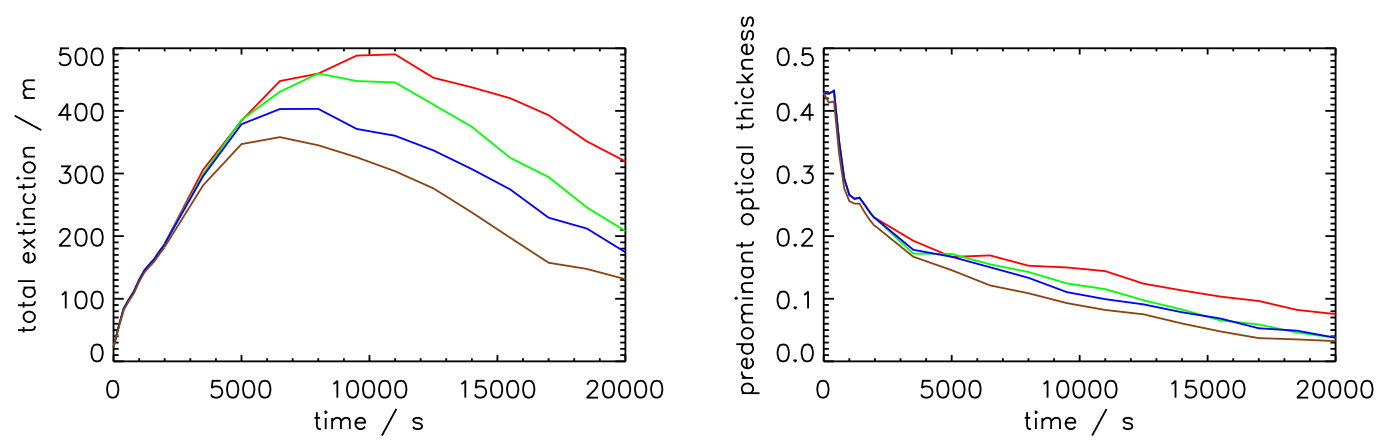

Fig. 9. Temporal evolution of total extinction and predominant optical thickness $\tau_{\text {pre }}$ for different thicknesses $L_{\mathrm{RH}}^{*}$ of the supersaturated layer. $L_{\mathrm{RH}_{i}^{*}}$ is $1 \mathrm{~km}$ (red), $800 \mathrm{~m}$ (green), $600 \mathrm{~m}$ (blue) resp. $400 \mathrm{~m}$ (brown). The ambient conditions are $T_{\mathrm{CA}}=217 \mathrm{~K}, \mathrm{RH}_{i}^{*}=120 \%$ and $s=0 \mathrm{~s}^{-1}$.

- heterogeneous nucleation thresholds $\mathrm{RH}_{\text {het }}$ for such soot particles in the range from $101 \%$ to $130 \%$. In a reference run the nucleation was turned off. To excite nucleation we introduced a synoptic-scale vertical motion $w_{0}$ in the total domain. The simulations are initialised with $T_{\mathrm{CA}}=217 \mathrm{~K}$, $\mathrm{RH}_{i}^{*}=120 \%$ and $s=0 \mathrm{~s}$. Between $t=1000 \mathrm{~s}$ and $3000 \mathrm{~s}$, a steady updraught with $w_{0}=10 \mathrm{~cm} \mathrm{~s}^{-1}$ leads to a $2 \mathrm{~K}$ temperature drop which corresponds to a rise of $\mathrm{RH}_{i}^{*}$ to $152 \%$. Between 4000s and 6000s the complete layer sinks with the same speed and finally reaches the original altitude. In Fig. 10 the mentioned time intervals are marked by vertical lines.

About $50 \%$ of the initial ice crystals $\mathcal{N}_{00}=3.4 \cdot 10^{12} \mathrm{~m}^{-1}$ survive the vortex phase (see UGS08). Thus at the beginning of the dispersion phase $\mathcal{N}_{0}=1.7 \cdot 10^{12} \mathrm{~m}^{-1}$ crystals and about the same number of soot particles are present. In the limiting case when all sublimated ice crystals re-nucleate in the course of the simulation, the ice crystal number would rise to the upper limit $\mathcal{N}_{00}$ (minus the number of crystals lost by sedimentation). Figure 10 shows the total number of ice crystals (i.e. sum of contrail ice and heterogeneously formed ice). First we discuss the reference case (black solid curve) where we turned off nucleation. The ice crystal number first drops, as crystals sublimate in the remnants of a sinking vortex pair. Between $t=1000 \mathrm{~s}$ and $4000 \mathrm{~s}$ the updraught speed is non-negative and no crystals sublimate. After 4000s subsidence $\left(w_{0}=-10 \mathrm{~cm} \mathrm{~s}^{-1}\right)$ leads to sublimation of crystals. At later stages the sedimentation process also contributes to the crystal loss. The other displayed quantities total extinction, optical thickness and effective radius depend on the depositional growth of the crystals. As expected they in/decrease during the up/downdraught period (with some delay).

With re-nucleation the evolution of $\mathcal{N}$ changes. The relative humidity in the vicinity of the contrail turns out to be mostly below $110 \%$, although the ambient value is $120 \%$. Hence new crystals form in the early dispersion phase only if their nucleation threshold is low, $\mathrm{RH}_{\text {het }} \leq 110 \%$. The ambient relative humidity rises during the updraught period and renucleation takes place for all $\mathrm{RH}_{\text {het }}$. Yet the conversion rate into crystals is probably small (if there is a pre-activation at all). For $\mathrm{RH}_{\text {het }} \geq 120 \%$ the contrail properties differ insignificantly from the reference case. If $\mathrm{RH}_{\text {het }}$ was only $101 \%$, $60 \%$ (over total time) and 10\% (during the updraught) of the soot particles would re-nucleate. The higher is $\mathrm{RH}_{\text {het }}$, the fewer crystals form. If crystals form at low nucleation thresholds they experience weak supersaturation, they hardly 

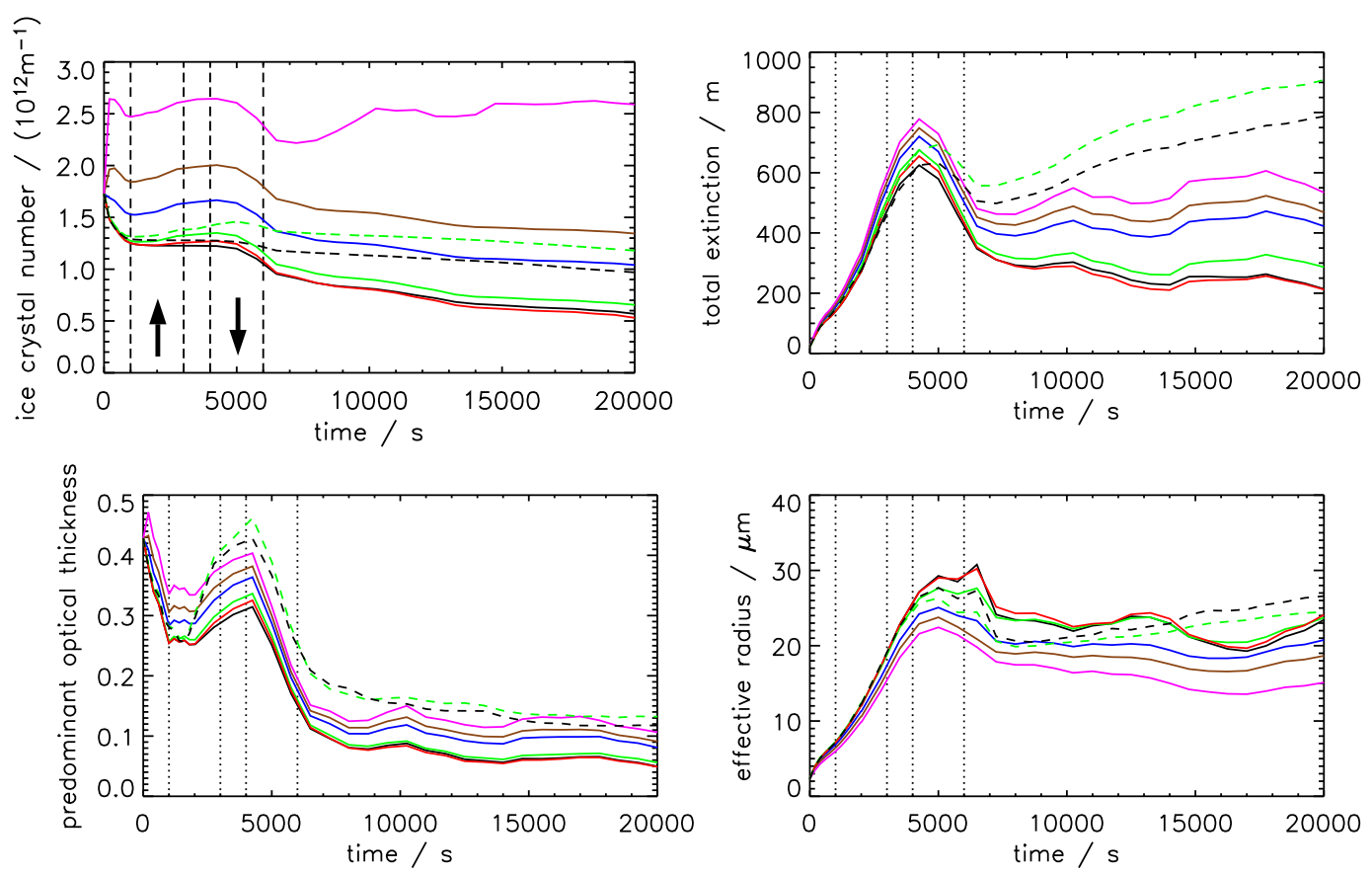

Fig. 10. Temporal evolution of total ice crystal number, total extinction, predominant optical thickness $\tau_{\text {pre }}$ and effective radius for different heterogeneous freezing thresholds $\mathrm{RH}_{\text {het }}=130 \%$ (red), 120\% (green), 110\% (blue), 105\% (brown) resp. 101\% (magenta). All quantities consider the contrail ice plus heterogeneously formed ice. In the reference simulation (black) nucleation was disabled. The dashed curves show runs with radiation switched on (black: reference run, green: $\mathrm{RH}_{\text {het }}=120 \%$ ). The vertical lines indicate the time intervals of different vertical motions $w_{0}$. The ambient conditions are initially $T_{\mathrm{CA}}=217 \mathrm{~K}, \mathrm{RH}_{i}^{*}=120 \%$ and $s=0 \mathrm{~s}^{-1}$.

affect the total ice mass, total extinction and effective radius until the updraught sets in. Only during the updraught period the re-nucleated crystals grow and lead to higher optical thicknesses, higher total extinction and smaller effective radii. These differences remain until the end of the simulation since the new crystals hardly sublimate during the downdraught phase.

In two more runs (dashed curves) we tested whether radiation-induced contrail lifting may enhance the heterogeneous nucleation process. We used again the cloudless summer day scenario where the radiation-induced contrail lifting is strong. The synoptic scale vertical motion is initialised as above. In a reference run no nucleation was allowed (black dashed curve). In a second run we set $\mathrm{RH}_{\text {het }}=120 \%$ (green dashed curve). The simulations without radiation showed that only a small fraction of pre-activated aerosols re-nucleate at this threshold humidity. Turning on the radiation routine leads to more nucleation events than in the two corresponding simulations with no radiation (compare black to green lines in Fig. 10). Nevertheless, the $\mathcal{N}$-evolution does not change qualitatively. Looking at the evolution of total extinction and optical thickness reveals that the additional nucleation is a second order effect compared to the general impact of radiation-induced contrail lifting. Clearly the reference run with radiation shows higher total extinction values than the "no radiation"-run with $\mathrm{RH}_{\text {het }}=101 \%$. It seems that radiation prolongs the contrail's lifetime, whereas we can neither deny nor confirm that heterogeneous nucleation process affects the lifetime.

\section{Discussion}

The sensitivity studies show that the initially present ice number concentration affects not only the contrail evolution up to the end of the vortex phase but later as well when the contrail evolves into a cirrus cloud. Under otherwise identical conditions a change of the initial crystal number strongly affects the crystal number present in the contrail-cirrus. This provides a handle to modify contrail-cirrus properties and lifetimes directly via the soot emission. The soot emission index, $E I_{\text {soot }}$ (i.e. the number of soot particles per $\mathrm{kg}$ fuel burnt), can vary over two orders of magnitudes for various aircraft $\left(10^{15} / \mathrm{kg}-10^{13} / \mathrm{kg}\right)$. A few $\mathrm{K}$ below the contrail formation threshold (Schmidt-Appleman criterion, see Schumann, 1996) each soot particle emitted nucleates an ice crystal (Kärcher and Yu, 2009). Hence reducing $E I_{\text {soot }}$ in a certain range (see Kärcher and $\mathrm{Yu}, 2009$, for details) reduces the initial ice crystal number in a linear fashion, and this will lead to a reduction of the optical thickness and lifetime of contrail-cirrus, a desired effect. A reduction of $E I_{\text {soot }}$ by two orders of magnitude can reduce the intrinsic timescale by one 
hour and the optical thickness by a factor 5 which both would reduce the climate impact. Hence future engines with lower soot number emissions could potentially lead to a reduction of the climate impact of aviation.

Radiative transfer studies of contrails with assumed properties (Myhre and Stordal, 2001; Stuber et al., 2006; Stuber and Forster, 2007) indicate that contrails contribute more to radiative forcing of climate during nighttime than during daytime although flights spend on average more time during day than during night. It might be that the degree of the day/night differences is actually smaller than computed in the pure radiative transfer studies. As our results show, contrail ice mass (hence optical effects) can grow due to the influence of radiation (i.e. radiative heating), which then also leads to a prolongation of their lifetime. In all our studies this effect was stronger for daytime than nighttime simulations. Thus, on average we expect that daytime contrails may be optically thicker and get older than nighttime contrails and such effects should be taken into account in comparisons of dayvs. nighttime contrails.

The strongest radiative heating of contrail-cirrus and therefore the strongest support of its further growth is given on summer days when no other clouds are beneath. Of course, these are the situations when contrail observation conditions from the ground (human observers and lidars) but also for visual and automated inspection of satellite imagery are optimal. It is quite possible therefore that observational data sets are biased in favour of the thicker contrails and contrailcirrus.

The radiative heating has an effect only after $1-2 \mathrm{~h}$ in our simulations, even when we assume a moist environment with $\mathrm{RH}_{i}^{*}=130 \%$ (contrail optical thickness $\sim 0.3$ ). Earlier contrail simulations that studied contrail evolution up to $30 \mathrm{~min}$ (Chlond, 1998; Gierens, 1996; Gierens and Jensen, 1998) found only weak (negligible) radiation influence which is conceivable when, as shown here, the radiation effects act only on timescales exceeding one hour. Accordingly, only Jensen et al. (1998), simulating up to $3 \mathrm{~h}$, found a radiative impact.

The depth of the supersaturated layer $L_{\mathrm{RH}_{i}^{*}}$ is not a limiting factor for the evolution of the contrail core as long as it exceeds $500 \mathrm{~m}$, since the contrail core region (with peaks in crystal number concentration and extinction) has a vertical extent of about $300 \mathrm{~m}$. Although the average depth of ice supersaturated layers seems to exceed $500 \mathrm{~m}$ (Spichtinger et al., 2003; Treffeisen et al., 2007; Rädel and Shine, 2007), the statistical distribution of the layer depths peaks at smaller values in all these studies and very thick ISSRs occur rarely. Hence, fallstreaks from contrails should rarely be as strong as in our simulations which generally assumed an ISSR layer depth of more than one $\mathrm{km}$.

\section{Conclusions}

From the sensitivity studies presented in this paper we can draw the following conclusions:

- The initial number of crystals, mostly equal to the number of soot particles emitted, has a direct impact on the microphysical and optical properties of contrails even in its later evolution into a cirrus cloud. Aviation could get "greener" if engines with reduced soot number emission indices were be introduced.

- Radiation can modify the contrail evolution qualitatively and can prolong the contrails' lifetimes under conditions of high supersaturation and/or temperature. The impact on the contrail evolution depends most sensitively on the existence of a cloud underneath, followed by the season and the time of day.

- Most favourable conditions for the contrail lifting and ice mass growth are given on cloudless summer days, the least favourable during cloudy winter nights. As otherwise cloud free skies present the optimum conditions for observations, this might produce a bias in statistics derived from observations.

- In a slightly stable atmosphere radiation can lead to 23 times higher total ice masses than in a strongly stable atmosphere.

- The weighting of nighttime vs. daytime contrails regarding their radiative forcing should be considered again taking into account that on average contrail cirrus might be optically thicker and longer lasting during the day than during night.

- Homogeneous nucleation of new crystals associated with a radiation-induced contrail lifting did not happen in our simulations.

- Ice nuclei (pre-activated soot cores) released from sublimating ice crystals during the vortex phase could lead to heterogeneous re-nucleation later under synoptic cooling conditions (uplifting), if the nucleation threshold is set to low values of $\mathrm{RH}_{\text {het }} \leq 105 \%$.

- The depth of the supersaturated layer $L_{\mathrm{RH}_{i}^{*}}$ is not a limiting factor for the contrail core evolution as long as it exceeds $500 \mathrm{~m}$. However, layer depth distributions for ice supersaturation layers peak at smaller values, hence fallstreaks from contrail-cirrus are expected to actually be weaker on average than in our numerical studies.

In this paper we have presented a large number of idealised studies of contrail-to-cirrus transition (see Table 1 for an overview). These have helped to acquire an improved understanding of the processes involved. For the future, however, 
we need certain steps away from the idealised scenarios towards more realistic situations. For instance, we have to take into account that contrails usually occur in clusters and interact over regions with heavy air traffic. It is probable that saturation effects will occur in air corridors (Gierens, 1998) that are not reflected in simulations of single contrails. Also, synoptic and mesoscale conditions have to be taken into account when statements are to be made on contrail-cirrus a couple of hours old. To enhance our model's capabilities in these directions is our current work.

Acknowledgements. The authors would like to thank B. Kärcher and U. Schumann for fruitful discussions, P. Spichtinger for help with the microphysics model, P. Smolarkiewicz and A. Dörnbrack for using/assisting with EULAG and C. Emde for several radiation simulations. We acknowledge U. Burkhardt for her careful reading of the manuscript.

This work contributes to the DLR/HGF-projects "Particles and Cirrus Clouds" (PAZI-2), "Climate-compatible air transport system" (CATS) and to the European Network of Excellence ECATS (Environmentally Compatible Air Transport System). The simulations have been carried out as a part of the special project "Ice-supersaturation and cirrus clouds" at the ECMWF.

Edited by: R. MacKenzie

\section{References}

Anderson, G., Clough, S., Kneizys, F., Chetwynd, J., and Shettle, E.: AFGL Atmospheric Constituent Profiles (0-120 km), AFGL (OPI), Hanscom AFB, MA 01736, AFGL-TR-86-0110, 1986.

Chlond, A.: Large-Eddy Simulation of Contrails, J. Atmos. Sci., 55, 796-819, 1998.

Corti, T., Luo, B. P., Fu, Q., Vömel, H., and Peter, T.: The impact of cirrus clouds on tropical troposphere-to-stratosphere transport, Atmos. Chem. Phys., 6, 2539-2547, 2006, http://www.atmos-chem-phys.net/6/2539/2006/.

Fu, Q.: An accurate parametrization of the solar radiative properties of cirrus clouds for climate models, J. Climate, 9, 2058-2082, 1996.

$\mathrm{Fu}, \mathrm{Q}$. and Liou, K.: Parametrization of the radiation properties of cirrus clouds, J. Atmos. Sci., 50, 2008-2025, 1993.

Fu, Q., Yang, P., and Sun, W.: An accurate parameterization of the infrared radiative properties of cirrus clouds for climate models, J. Climate, 11, 2223-2237, 1998.

Fusina, F., Spichtinger, P., and Lohmann, U.: Impact of ice supersaturated regions and thin cirrus on radiation in the midlatitudes, J. Geophys. Res., 112, D24S14, doi:10.1029/2007JD008449, 2007.

Gierens, K.: The Influence of Radiation on the Diffusional Growth of Ice Crystals, Beiträge zur Physik der Atmosphäre, 67, 181193, 1994.

Gierens, K.: Numerical Simulations of Persistent Contrails, J. Atmos. Sci., 53, 3333-3348, 1996.

Gierens, K.: How the Sky Gets Covered with Condensation Trails, Meteorol. Z., 7, 181-187, 1998.
Gierens, K. and Jensen, E.: A numerical study of the contrail-tocirrus transition, Geophys. Res. Lett., 25, 4341-4344, 1998.

Huebsch, W. and Lewellen, D.: Sensitivity Study on Contrail Evolution, 36th AIAA Fluid Dynamics Conference and Exhibit, AIAA, 2006-3749, 2006.

Jensen, E., Ackerman, A., Stevens, D., Toon, O., and Minnis, P.: Spreading and growth of contrails in a sheared environment, J. Geophys. Res., 103, 31557-31568, 1998.

Kärcher, B. and Yu, F.: Role of aircraft soot emissions in contrail formation, Geophys. Res. Lett., 36, L01804, doi:10.1029/ 2008GL036649, 2009.

Kärcher, B., Busen, R., Petzold, A., Schröder, F., Schumann, U., and Jensen, E.: Physicochemistry of aircraft-generated liquid aerosols, soot, and ice particles, 2. Comparison with observations and sensitivity studies, J. Geophys. Res., 103, 17129-17148, 1998.

Liu, H., Wang, P., and Schlesinger, R.: A Numerical Study of Cirrus Clouds. Part II: Effects of Ambient Temperature, Stability, Radiation, Ice Microphysics, and Microdynamics on Cirrus Evolution, J. Atmos. Sci., 60, 1097-1119, 2003.

Myhre, G. and Stordal, F.: On the tradeoff of the solar and thermal infrared radiative impact of contrails, Geophys. Res. Lett., 28, 3119-3122, 2001.

Rädel, G. and Shine, K.: Evaluation of the use of radiosonde humidity data to predict the occurrence of persistent contrails, Q. J. Roy. Meteor. Soc., 133, 1413-1423, 2007.

Schumann, U.: On conditions for contrail formation from aircraft exhausts, Meteorol. Z., NF, 5, 4-23, 1996.

Schumann, U., Ström, J., Busen, R., Baumann, R., Gierens, K., Krautstrunk, M., Schröder, F., and Stingl, J.: In situ observations of particles in jet aircraft exhausts and contrails for different sulfur-containing fuels, J. Geophys. Res., 101, 6853-6870, 1996.

Schumann, U., Arnold, F., Busen, R., Curtius, J., Kärcher, B., Kiendler, A., Petzold, A., Schlager, H., Schröder, F., and Wohlfrom, K.: Influence of fuel sulfur on the composition of aircraft exhaust plumes: The experiments SULFUR 1-7, J. Geophys. Res., 107, doi:10.1029/2001JD000813, 2002.

Smolarkiewicz, P. and Margolin, L.: On Forward-in-Time Differencing for Fluids: an Eulerian/Semi-Lagrangian NonHydrostatic Model for Stratified Flows, Numerical Methods in Atmospheric and Oceanic Modelling: The André J. Robert Memorial Volume, 1997.

Smolarkiewicz, P. and Margolin, L.: MPDATA: A Finite-Difference Solver for Geophysical Flows, J. Comput. Phys., 140, 459-480, 1998.

Spichtinger, P. and Gierens, K. M.: Modelling of cirrus clouds Part 1a: Model description and validation, Atmos. Chem. Phys., 9, 685-706, 2009, http://www.atmos-chem-phys.net/9/685/2009/.

Spichtinger, P., Gierens, K., Leiterer, U., and Dier, H.: Ice supersaturation in the tropopause region over Lindenberg, Germany, Meteorol. Z., 12, 143-156, 2003.

Stuber, N. and Forster, P.: The impact of diurnal variations of air traffic on contrail radiative forcing, Atmos. Chem. Phys., 7, 3153-3162, 2007, http://www.atmos-chem-phys.net/7/3153/2007/. 
Stuber, N., Forster, P., Radel, G., and Shine, K.: The importance of the diurnal and annual cycle of air traffic for contrail radiative forcing, Nature, 441, 864-867, 2006.

Treffeisen, R., Krejci, R., Ström, J., Engvall, A. C., Herber, A., and Thomason, L.: Humidity observations in the Arctic troposphere over Ny-Ålesund, Svalbard based on 15 years of radiosonde data, Atmos. Chem. Phys., 7, 2721-2732, 2007,

http://www.atmos-chem-phys.net/7/2721/2007/.
Unterstrasser, S. and Gierens, K.: Numerical simulations of contrail-to-cirrus transition - Part 1: An extensive parametric study, Atmos. Chem. Phys., Atmos. Chem. Phys., 10, 2017-2036, 2010 ,

http://www.atmos-chem-phys.net/10/2017/2010/.

Unterstrasser, S., Gierens, K., and Spichtinger, P.: The evolution of contrail microphysics in the vortex phase, Meteorol. Z., 17, 145-156, 2008. 